\title{
Atypical Antipsychotic Augmentation Strategies in the Context of Guideline-based Care for the Treatment of Major Depressive Disorder
}

\author{
Ashwin A. Patkar · Chi-Un Pae
}

Published online: 25 May 2013

(C) Springer International Publishing Switzerland 2013

\begin{abstract}
There is a growing body of evidence that supports the use of atypical antipsychotics as augmentation agents for nonpsychotic unipolar major depressive disorder (MDD) in adults. Unfortunately, varying definitions of treatment-resistant depression, the limited evidence available for interventions after two or more treatment failures, and when and whether to use medications from nonantidepressant classes, remain a key gap in the knowledge base for clinicians. We identified and reviewed the following guidelines to discuss the status of augmentation therapy with atypical antipsychotic agents in MDD: American Psychiatric Association practice guidelines for treatment of patients with MDD; Canadian Network for Mood and Anxiety Treatments clinical guidelines for the management of MDD in adults; National Institute for Health and Clinical Excellence guidelines for treatment and management of depression in adults; British Association of Psychopharmacology guidelines for treatment of depressive disorders; Institute for Clinical Systems Improvement healthcare guideline for MDD in adults in primary care; clinical practice recommendations for depression; international consensus statement on MDD; German Society of Psychiatry, Psychotherapy and Neurology guidelines for unipolar depression; and World Federation of Societies of
\end{abstract}

Previous Presentation: Part of the information presented in this article was presented at the round table discussion of psychiatrists from Asia and the USA, sponsored by Korea Otsuka International Asia and Arab.

A. A. Patkar $(\bowtie)$. C.-U. Pae

Department of Psychiatry and Behavioral Sciences,

Duke University, Durham, North Carolina, USA

e-mail: ashwin.patkar@duke.edu

C.-U. Pae

Department of Psychiatry, The Catholic University of Korea

College of Medicine, Seoul, Republic of Korea
Biological Psychiatry guidelines for biological treatment of unipolar depressive disorders in primary care. Reflecting the cumulative evidence in the past decade, augmentation strategies including atypical antipsychotic augmentation are recommended in most guidelines for partial or nonresponders, at the same stage as switching or combination strategies. However, there are few direct comparisons of different augmentation strategies and little information about the optimal duration of augmentation strategies or use in special populations. Clinicians should note that guidelines are derived from an evolving database of evidence and cannot take into account the myriad of clinical variables that differ between individual patients. Therefore, they are intended to provide a useful framework for the management of depression and should be used in conjunction with other recognized sources of patient information and the application of clinical wisdom.

\section{Introduction}

Despite the availability of new and effective treatments for major depressive disorder (MDD) over the past 20 years, evidence in practice settings continues to demonstrate high rates of inadequate antidepressant treatment in terms of dose and duration, inappropriate changes in treatment, as well as low adherence and high dropout rates, all contributing to low rates of remission for MDD [1]. Clearly there is a need for more informed clinical decision-making that has the potential to improve outcomes. Clinical practice guidelines are systematically developed statements to assist practitioner and patient decisions about appropriate healthcare for specific clinical circumstances [2, 3]. In addition, guidelines can play an important role in health policy formation and have evolved to cover topics across the healthcare continuum (e.g. screening and diagnosis). 
The potential benefits of guidelines are only as good as the quality of the guidelines themselves. Appropriate methodologies and rigorous strategies in the guideline development process are important for the successful implementation of the resulting recommendations [4].

For the substantial number of patients with MDD who fail to respond to the initial antidepressant trial, the clinical question is: what is the next step in treatment? Although many guidelines exist to aid in the initial management of MDD, recommendations for treatment-resistant depression are more limited. There is a growing body of evidence that supports the use of atypical antipsychotics as augmentation agents for nonpsychotic unipolar MDD in adults [5]. Two atypical antipsychotic agents, aripiprazole and extended release quetiapine, have been approved for adjunctive therapy in adult patients with MDD who show inadequate response to antidepressants in the USA. In addition, olanzapine plus fluoxetine has also been approved for treatment-resistant depression in the USA. Unfortunately, varying definitions of treatment-resistant depression, the limited evidence available for interventions after two or more treatment failures, and when and whether to use medications from nonantidepressant classes, remain a key gap in the evidence base leaving clinicians uncertain as to how to proceed with their patients with treatment-resistant depression. We identified and reviewed the following guidelines to discuss the status of augmentation therapy with atypical antipsychotic agents in MDD: American Psychiatric Association Practice Guidelines for treatment of patients with MDD [6, 7]; Canadian Network for Mood and Anxiety Treatments (CANMAT) clinical guidelines for the management of MDD in adults [8]; National Institute for Health and Clinical Excellence (NICE) guidelines for treatment and management of depression in adults [9]; British Association of Psychopharmacology (BAP) guidelines for treatment of depressive disorders [10]; Institute for Clinical Systems Improvement (ICSI) healthcare guideline for MDD in adults in primary care [11]; clinical practice recommendations (CPR) for depression [12]; international consensus statement (ICS) on MDD [13]; the German Society of Psychiatry, Psychotherapy and Neurology (DGPPN) guidelines for unipolar depression [14]; and World Federation of Societies of Biological Psychiatry (WFSBP) guidelines for biological treatment of unipolar depressive disorders in primary care [15].

\section{American Psychiatric Association Guidelines}

\subsection{Guideline Development}

In October 2010, the American Psychiatric Association (APA) released the 3rd edition of practice guidelines for the treatment of patients with major depressive disorder [16]. The development process is detailed in a document titled 'APA Guideline Development Process', which is available from the APA Department of Quality Improvement and Psychiatric Services. Key features of this process included: a comprehensive literature review of over 13,000 publications to identify all relevant randomized clinical trials as well as less rigorously designed clinical trials and case series, development of evidence tables that reviewed the key features of each identified study, initial drafting of the guideline by a work group that included psychiatrists with clinical and research expertise in MDD, production of multiple revised drafts with widespread review from stakeholders, review of the final draft by an independent review panel of experts with no relationships with industry, and final approval by the APA assembly and board of trustees. The APA plans to revise the guidelines at regular intervals with availability of new evidence. The recommendations are based on the best available data and expert consensus with regard to clinical decision making. The summary of treatment recommendations outlines the level of confidence with which each recommendation is made (level 1: substantial clinical confidence; level 2: moderate clinical confidence; level 3: individual circumstances).

\subsection{Choice of an Initial Treatment Modality}

The APA guidelines recommend that treatment in the acute phase should be aimed at inducing remission of the MDD episode and achieving a return to the patient's baseline level of functioning. Acute phase treatment may include antidepressant pharmacotherapy, depression-focused psychotherapy, the combination of medications and psychotherapy, or other somatic therapies such as electroconvulsive therapy (ECT). The APA specifies that 4-8 weeks of adequate treatment are needed before concluding that a patient is partly responsive or unresponsive to an initial intervention.

\subsection{Strategies to Address Nonresponse to Initial Antidepressant Treatment}

For individuals who have not responded fully to the initial antidepressant, the guidelines recommend that diagnosis should be reappraised, treatment adherence and side effects assessed, complicating co-occurring conditions and psychosocial factors reviewed, and the treatment adjusted accordingly. A measurement-based approach using validated rating scales is recommended to monitor improvement. Treatment changes include optimizing the medication dose if the upper limit of a medication dose has not been reached, or changing to another antidepressant, augmenting the antidepressant with a depression-focused psychotherapy or with other pharmacological agents or considering ECT. 


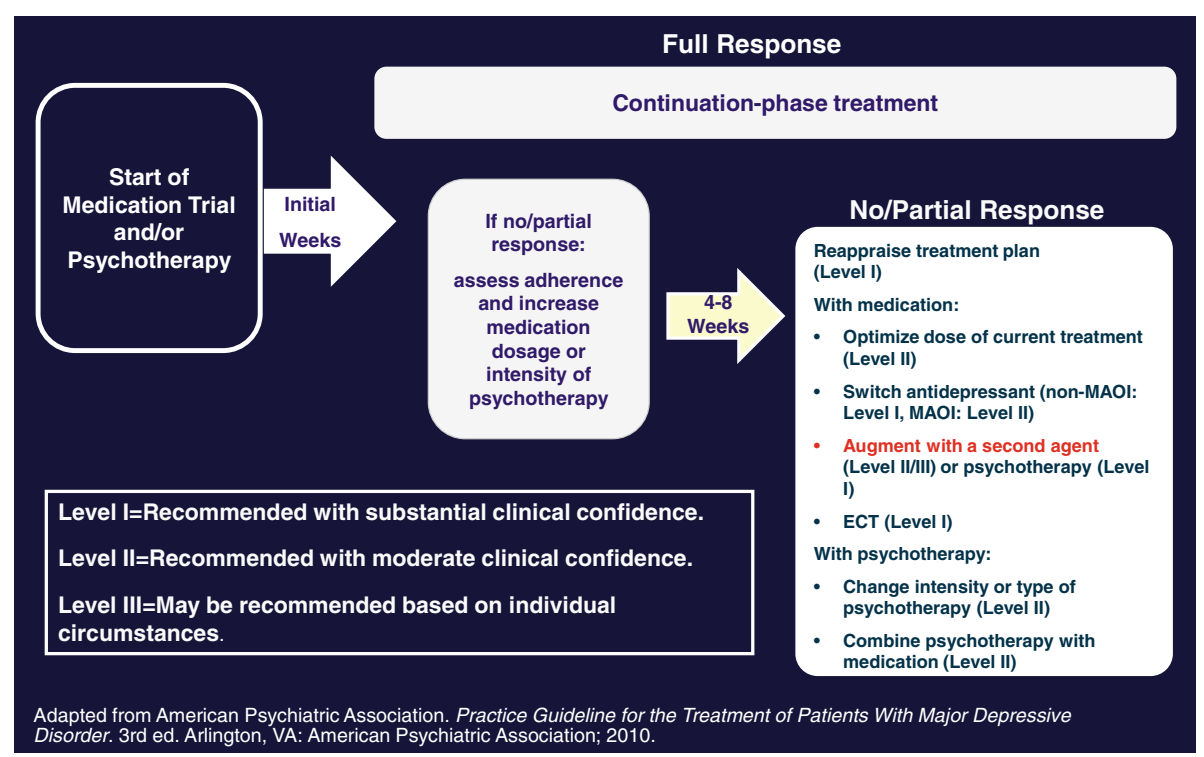

Fig. 1 Revised American Psychiatric Association guidelines for the acute-phase treatment of major depressive disorder. ECT electroconvulsive therapy, MAOI monoamine oxidase inhibitor. Adapted from American Psychiatric Association [16]

Fig. 1 summarizes APA guidelines for initial and subsequent treatment approaches for MDD.

\subsection{Augmentation Strategies}

Augmentation of antidepressant medications can utilize another non monoamine oxidase inhibitor antidepressant (level 2), generally from a different pharmacological class, or a nonantidepressant medication such as lithium (level 2), thyroid hormone (level 2), or an atypical antipsychotic (level 2). Additional strategies (level 3) with less evidence for efficacy include augmentation using an anticonvulsant, omega-3 fatty acids, folate, psychostimulants, buspirone and benzodiazepines. Table 1 summarizes the different augmenting agents and their level of confidence.

Although the APA guidelines indicate the same level of confidence (level 2) for augmentation with atypical antipsychotics, lithium, thyroid hormone or another antidepressant, it is worth noting that among these agents, atypical antipsychotics have been studied most systematically with the largest number of randomized controlled trials and well defined, treatment-resistant samples. The meta-analysis of 16 placebo controlled, randomized clinical trials of atypical antipsychotic augmentation therapy for patients with MDD $(N=3,480)$ showed that augmentation with an atypical antipsychotic agent was significantly more effective than placebo in terms of rates of response [odds ratio $(\mathrm{OR})=$ 1.69, $95 \%$ confidence interval (CI) 1.46 to 1.95 , $p<0.00001]$ and remission $(\mathrm{OR}=2.00,95 \% \mathrm{CI} 1.69$ to 2.37, $p<0.00001$ ) [5]. Although aripiprazole and quetiapine (olanzapine plus fluoxetine, approved for treatment-resistant depression) have received approval as an adjunct to
Table 1 Revised American Psychiatric Association guidelines: augmentation recommendations for no/partial response to antidepressant therapy

\begin{tabular}{ll}
\hline Augmentation options & $\begin{array}{l}\text { Level of clinical } \\
\text { confidence }(1-3)\end{array}$ \\
\hline
\end{tabular}

\begin{tabular}{ll}
\hline Psychotherapy & 1 \\
Second antidepressant & 2 \\
Atypical antipsychotic & 2 \\
Thyroid hormone & 2 \\
Mood stabilizer & 2 \\
Anticonvulsant & 3 \\
Psychostimulant & 3 \\
Omega-3 fatty acid & 3 \\
Folic acid & 3 \\
Anxiolytic or sedative/hypnotic & 3
\end{tabular}

Level 1 Recommended with substantial clinical confidence

Level 2 Recommended with moderate clinical confidence

Level 3 May be recommended on the basis of individual circumstances

Adapted from American Psychiatric Association. practice guideline for the treatment of patients with major depressive disorder, 2010 [16]

antidepressant medications in the USA, this meta-analysis also showed efficacy for risperidone and olanzapine. Discontinuation rates for adverse effects were also higher in the active augmentation groups compared with placebo $(\mathrm{OR}=3.91,95 \% \mathrm{CI} 2.68$ to 5.72, $p<0.00001)$, suggesting that such effects need to be taken into consideration when choosing to augment antidepressant response with an 
atypical antipsychotic agent [17]. Compared to the evidence for atypical antipsychotics, the evidence for other level 2 augmenting agents is less robust. For example, the evidence for lithium or triiodothyronine (T3) augmentation is primarily derived from trials of tricyclic antidepressants in which treatment resistance of the sample was not well established. There is limited empirical support for lithium or $\mathrm{T} 3$ augmentation of newer antidepressants in patients who have failed two or more antidepressant trials. Similarly, the evidence for augmentation with another antidepressant comes from studies with small sample sizes [18] or from trials that have combined antidepressants from the beginning [19]. The APA guidelines consider psychostimulants as level 3 augmenting agents that lack empirical evidence for use in clinical practice with a high level of confidence. To date, few data from controlled studies address the longer term efficacy or side effects of atypical antipsychotic augmentation.

\section{Canadian Network for Mood and Anxiety Treatments Guidelines}

\subsection{Guideline Development}

CANMAT collaborated on the publication of evidencebased clinical guidelines for the treatment of depressive disorders in 2001. These guidelines were revised by CANMAT in August 2009 based on new evidence [8]. The development process included a systematic literature review, creating evidence tables to specify level of evidence and grading recommendations according to the line of treatment. A first-line treatment recommendation represents a balance of efficacy, tolerability and clinical support. Clinical support refers to the application of expert opinion of the CANMAT committees to ensure that evidence-supported interventions are realistic and applicable for clinical practice. Second-line and third-line treatments are reserved for situations in which first-line treatments are not indicated or cannot be used, or when first-line treatments have failed or have tolerability issues.

\subsection{Choice of an Initial Treatment Modality}

Similar to the APA guidelines, CANMAT guidelines emphasize the importance of a thorough diagnostic assessment to determine the appropriate initial treatment modality and self or clinician-rated scales to monitor response. The guidelines point out that pharmacotherapy remains the most studied and best evidenced treatment for MDD [20]; however, clinicians may choose from a variety of treatment options including psychotherapy depending on individual circumstances. Selective serotonin reuptake inhibitors, selective serotonin/norepinephrine reuptake inhibitors and bupropion are recommended as first-line antidepressants for initial treatment. Treatment may be changed as early as 2 weeks if there is little improvement with antidepressant use (e.g. $\leq 20 \%$ improvement in scores on a depression rating scale). Patients showing more than minimal improvement (e.g. $\geq 20 \%$ improvement in scores on a depression rating scale) after 4-6 weeks should continue to receive the antidepressant for another 2-4 weeks before considering additional strategies.

\subsection{Strategies to Address Nonresponse to Initial Antidepressant Treatment}

When there has been no improvement following an adequate antidepressant trial the clinician should re-evaluate diagnostic and compliance issues. Treatment options include adding evidence-based psychotherapy [21], switching to a neurostimulation treatment such as ECT [8], or continuing with pharmacological strategies [20]. Pharmacological strategies include switching to a different antidepressant monotherapy, or adding another agent to the first antidepressant. The CANMAT guidelines use the general term 'add-on' instead of 'augmentation' and 'combination' because of difficulties distinguishing between additions with and without intrinsic antidepressant activity.

\subsection{Augmentation Strategies}

The CANMAT guidelines consider add-on strategies to be among the best validated pharmacological treatments for treatment-resistant depression. Table 2 summarizes the add-on pharmacological strategies following failure of initial antidepressants.

There is level 1 evidence to support add-on treatment with some atypical antipsychotics and lithium for patients who have inadequate response to the initial antidepressant, and level 2 support for T3 and bupropion. There is level 3 evidence but also negative studies with buspirone, other antidepressants, methylphenidate, modafinil and pindolol, so these agents are not recommended as first or second-line treatments. Unlike the APA guidelines, the CANMAT guidelines differentiate between individual atypical antipsychotics in terms of recommendation as add-on agents. Aripiprazole (level 1 evidence), olanzapine (level 1 evidence) and risperidone (level 2 evidence) are recommended as first-line agents, quetiapine (level 2 evidence) is recommended as second-line, and ziprasidone (level 3 evidence) is considered a third-line agent. The guidelines suggest that the doses of atypical antipsychotics used as add-on treatment for MDD should be usually lower than used for mania or schizophrenia and the side effects of these agents, especially weight gain, the potential for metabolic syndrome and the risk of extrapyramidal side 
Table 2 Recommendations for nonresponse and incomplete response to an initial antidepressant from the Canadian Network for Mood and Anxiety Treatments guideline

\begin{tabular}{|c|c|c|}
\hline \multirow[t]{4}{*}{ First line } & Add-on another agent & Aripiprazole (level 1) \\
\hline & & Lithium (level 1) \\
\hline & & Olanzapine (level 1) \\
\hline & & Risperidone (level 2) \\
\hline \multirow[t]{5}{*}{ Second line } & Add-on another agent & Bupropion (level 2) \\
\hline & & $\begin{array}{l}\text { Mirtazapine/mianserin } \\
\quad \text { (level 2) }\end{array}$ \\
\hline & & Quetiapine (level 2) \\
\hline & & $\begin{array}{l}\text { Triiodothyronine } \\
\text { (level 2) }\end{array}$ \\
\hline & & $\begin{array}{l}\text { Other antidepressants } \\
\text { (level 3) }\end{array}$ \\
\hline \multirow[t]{4}{*}{ Third line } & Add-on another agent & Buspirone (level 3) \\
\hline & & Modafinil (level 2) \\
\hline & & Ziprasidone (level 3) \\
\hline & & Stimulants (level 3) \\
\hline \multicolumn{3}{|c|}{ Level of evidence: } \\
\hline 1 & $\begin{array}{l}\text { At least two randomized, controlled clinical trials } \\
\text { with adequate sample sizes, preferably placebo } \\
\text { controlled, and/or meta-analysis with narrow } \\
\text { confidence intervals }\end{array}$ & \\
\hline 2 & $\begin{array}{l}\text { At least one randomized, controlled clinical trial with } \\
\text { adequate sample size and/or meta-analysis with wide } \\
\text { confidence intervals }\end{array}$ & \\
\hline 3 & $\begin{array}{l}\text { Nonrandomized, controlled prospective studies or } \\
\text { case series or high-quality retrospective studies }\end{array}$ & \\
\hline 4 & Expert opinion/consensus & \\
\hline \multicolumn{3}{|c|}{ Line of treatment: } \\
\hline First line: & Level 1 or level 2 evidence, plus clinical support & \\
\hline Second line: & Level 3 evidence or higher, plus clinical support & \\
\hline Third line: & Level 4 evidence or higher, plus clinical support & \\
\hline
\end{tabular}

From Kennedy et al. [8]

effects, must be considered in the risk-benefit assessment, particularly in the context of long-term therapy.

\section{Augmentation Strategies in Other Guidelines}

The NICE guidelines for treatment of MDD were developed by the National Collaborating Center for Mental Health in the UK and published in October 2009 [9]. The NICE guidelines recommend augmentation as a treatment option after initial antidepressant treatment for MDD is not successful. The guidelines consider atypical antipsychotics such as aripiprazole, quetiapine, risperidone and olanzapine as well as lithium and other antidepressants such as mirtazapine and mianserin to be appropriate augmenting agents. Clinicians are asked to monitor metabolic parameters, weight, extrapyramidal side effects and prolactin (for risperidone) while using atypical antipsychotics. At the time the NICE guidelines were published, atypical antipsychotics were not approved as augmentation agents for MDD in the UK. Nevertheless, they are recommended for augmentation therapy based on the available evidence. Agents such as T3, anticonvulsants, pindolol, benzodiazepines ( $>2$-week treatment) and buspirone are not recommended for routine use for augmentation due to insufficient evidence. BAP published a revised set of evidence-based guidelines to treat depressive disorders following an expert consensus meeting in 2006 [10]. Augmentation is considered a next-step treatment following partial/insufficient treatment response to the current antidepressant or after switching strategies have been unsuccessful. BAP defined the different categories of recommendation as follows: A, directly based on category I evidence; B, directly based on category II evidence or extrapolated recommendation from category I evidence; C, directly based on category III evidence or extrapolated recommendation from category I or II evidence; D, directly based on category IV evidence or extrapolated recommendation from categories I, II or III evidence; and S, standard of good practice. Lithium and olanzapine are supported by a category A recommendation, 
while aripiprazole, quetiapine, risperidone, mirtazapine and T3 carry a category B recommendation. Category $\mathrm{C}$ recommendations include psychostimulants and anticonvulsants. It must be noted that the BAP guidelines were formulated before the current body of evidence for aripiprazole and quetiapine was available [22-24]. The ICSI healthcare guidelines were developed for the treatment of major depression in primary care in the USA [11]. Augmentation therapy is recommended for situations in which the patient's depression is either partly responsive to treatment $(<25 \%$ improvement after at least 6 weeks of antidepressant at a therapeutic dose) or is treatment resistant (defined as at least three separate antidepressant treatment trial failures). The ICSI guidelines suggest consultation or referral to a psychiatrist for augmentation therapy. Recommended augmentation agents include lithium, T3, atypical antipsychotics and antidepressants (bupropion, buspirone and mirtazapine). Among atypical antipsychotics, aripiprazole is considered to have high quality evidence; however, it is noted that meta-analyses have not found differences in efficacy between atypical antipsychotics $[5,25,26]$. The CPR for depression was published in Australia [12]. Therapeutic strategies to manage partial or nonresponse to initial antidepressant treatment include dose optimization, augmentation, combination, substitution (switching) or ECT. The level of the evidence within the depression CPR has been rated according to the National Health and Medical Research Council. Levels of evidence criteria-recommended pharmacological augmentation options are lithium (level 1), atypical antipsychotics (level 1), short-term benzodiazepine use (level 1) or thyroid hormone (level not defined). The CPR state that there is little controlled evidence to support antidepressant combinations; however, the strategy may be used clinically to treat nonresponse. A series of articles by an international consensus group outlined depression treatment guidelines from China, Japan, the Middle East, America and Europe [13, 27-30]. To avoid duplicating previous guidelines, the consensus group decided to adapt Japan's existing treatment algorithm for depression, and released the ICS on MDD in an attempt to merge evidence base and standardize clinical practice for the treatment of MDD across different regions of the world [13]. For partial responders to the initial 6-8-week antidepressant trial, the ICS recommends antidepressant dose modification, switching to a different antidepressant, or augmentation/ combination strategies. Augmentation/combination strategies include, but are not limited to, lithium, atypical antipsychotics, T3, mirtazapine, mianserin, or bupropion.

Some guidelines are more circumspect in recommending atypical antipsychotic agents for augmentation therapy in MDD. For example, the German guidelines compiled by the DGPPN [14] recommend augmentation by lithium (evidence level B, strength of evidence Ia) rather than switching or combination strategies if 4-6 weeks of adequate antidepressant therapy produces insufficient response. Atypical antipsychotics are not routinely recommended as augmentation agents for MDD by DGPPN, but are reserved for patients with psychotic depression. Lithium is also recommended as the first augmentation option before considering atypical antipsychotics, thyroid hormone or buspirone for nonresponders or partial responders in the WFSBP guidelines for biological treatment of unipolar depressive disorders in primary care [15]. The relatively less emphasis placed on atypical antipsychotics for augmentation in the WFSBP guidelines may reflect the body of evidence available at the time the guidelines were developed.

\section{Does Adherence to Guideline-based Care Improve Outcomes in Major Depressive Disorder?}

Data derived from older studies have found improved outcomes with guideline-based care, in particular models of collaborative care for depression in primary care [31]. An algorithm-based care for MDD (ALGO) based on the Texas Medication Algorithm Project in the USA [32] was compared to treatment as usual (TAU) in 12 clinics over 12 months. The ALGO intervention was associated with statistically and clinically significantly better clinical outcomes than TAU in the primary (and most secondary) efficacy assessments, including the Inventory of Depressive Symptoms Clinical rated (IDS-C30) and self-rated versions (IDS-SR30). The magnitude of the difference between ALGO and TAU was robust (mean IDS-C30 difference 4.5 points; mean IDS-SR30 difference 7.5 points). The significant advantage for ALGO was seen in the first quarter, with no evidence that TAU patients caught up with their ALGO counterparts during the ensuing 9-month period. A Japanese trial [33] found a four-step algorithm-guided treatment (AGT) to be superior to TAU in remission rates (AGT 60.2\%, TAU 49.7\%) and the median number of days to achieve remission (AGT group 93 days, TAU group 191 days, hazard ratio $=1.5,95 \%$ CI 1.2 to 1.8 ). The authors suggested that a higher rate of lithium augmentation in the AGT group (20.5\%) compared to the TAU group $(4.7 \%)$ may have led to the greater remission rate. Favourable results have also been consistently observed for the collaborative-care model, an evidence-based practice that involves a multidisciplinary depression care team (e.g. primary care providers, nurse care managers, pharmacists, psychologists, psychiatrists) providing guideline-concordant depression treatment in the primary care setting [34, 35]. To date, there have been no controlled trials comparing TAU against guideline-driven care that has included augmentation therapy with atypical antipsychotics as a treatment option. 


\begin{abstract}
Several sets of practice guidelines for MDD have been developed: APA, CANMAT, NICE, BAP, ICSI, CPR, ICS, DGPPN and WFSBP

All emphasize remission as the treatment goal, a measurement-based approach to guide care, and acute as well as maintenance treatment for MDD.

Initial treatment is antidepressant trial and/or psychotherapy.

Augmentation, switching or combination or psychotherapy are recommended as appropriate treatment options following failure of initial treatment or for treatmentresistant depression.

Lithium, atypical antipsychotics, T3 and certain antidepressants are recommended as pharmacological augmenting agents with good evidence in most guidelines.

Most guidelines recommend atypical antipsychotics as a class for augmentation therapy; however, some guidelines (e.g. CANMAT, BAP) recommend individual atypical agents.

All guidelines recommend monitoring side effects of augmenting agents for riskbenefit assessment, especialiy in the context of long-term therapy.
\end{abstract}

Fig. 2 Summary of recent guidelines for major depressive disorder. APA American Psychiatric Association, BAP British Association of Psychopharmacology, CANMAT Canadian Network for Mood and Anxiety Treatments, $C P R$ clinical practice recommendation, $D G P P N$ German Society of Psychiatry, Psychotherapy and Neurology, ICS

\section{Conclusions}

In the past few years, several guidelines have been developed to provide practical recommendations for the management of adult patients with MDD. The guidelines are a combination of data-driven evidence, clinical expertise and opinion from different parts of the world. Despite some differences that are only to be expected, there are broad areas of agreement in approaches for managing MDD. Fig. 2 summarizes some of the key aspects of the treatment of MDD. Reflecting the cumulative evidence in the past decade, augmentation strategies including atypical antipsychotic augmentation, are recommended in most guidelines for partial or nonresponders, at par with switching or combination strategies. However, there are few direct comparisons of different augmentation strategies, and little information about the optimal duration of augmentation strategies or use in special populations (e.g. adolescents or elderly people). Clinicians should note that guidelines are derived from an evolving database of evidence and cannot international consensus statement, ICSI Institute for Clinical Systems Improvement, $M D D$ major depressive disorder, NICE National Institute for Health and Clinical Excellence, T3 triiodothyronine, WFSBP World Federation of Societies of Biological Psychiatry

take into account the myriad of clinical variables that differ between individual patients. Therefore, they are intended to provide a useful framework for the management of depression and should be used in conjunction with other recognized sources of patient information and the application of clinical wisdom.

Most individuals with depression go untreated or undertreated. A recent large study in the USA found only $21 \%$ of individuals in the sample with a diagnosis of MDD had received at least one form of APA guideline-concordant therapy in the previous year [36]. Clearly, more research is needed to refine our understanding of the best strategies for implementing guideline-based care [37], and improve the consistency of care provided for patients with MDD in busy, 'real-world' practices. If successful, this approach will ultimately benefit patients by improving outcomes.

Acknowledgments Although Korea Otsuka International Asia and Arab (KOIAA) was involved in supporting the production of the 
supplement, the content of the manuscript, its review and revision, and the decision to submit to CNS Drugs were made solely by the authors and the supplement guest editor.

Sources of Financial Support The authors did not receive honorarium for writing this manuscript.

Conflict of interest Dr Patkar is a consultant for Bristol-Myers Squibb, GlaxoSmithKline and Reckitt Benckiser; is on the speaker's bureau of Bristol-Myers Squibb, GlaxoSmithKline and Reckitt Benckiser; and has received research support from National Institutes of Health, AstraZeneca, Bristol-Myers Squibb, Forest, GlaxoSmithKline, Janssen, McNeil Consumer and Specialty Inc, Organon, Jazz Pharmaceuticals and Pfizer. Dr Pae has received research grants from Eisai Korea, Janssen Pharmaceuticals Korea, Korean Health Technology R\&D Project, Ministry of Health \& Welfare, Republic of Korea and Korean Research Foundation; and has received honoraria from Pfizer Korea and Otsuka Korea.

Disclosure This manuscript has been published in a journal supplement that was created with an unrestricted educational grant from Korea Otsuka International Asia and Arab (KOIAA).

\section{References}

1. Kessler RC, Berglund P, Demler O, Jin R, Koretz D, Merikangas $\mathrm{KR}$, et al. The epidemiology of major depressive disorder: results from the National Comorbidity Survey Replication (NCS-R). JAMA. 2003;289(23):3095-105.

2. Woolf SH, Grol R, Hutchinson A, Eccles M, Grimshaw J. Clinical guidelines: potential benefits, limitations, and harms of clinical guidelines. BMJ. 1999;318(7182):527-30.

3. Schulberg HC, Katon W, Simon GE, Rush AJ. Treating major depression in primary care practice: an update of the Agency for Health Care Policy and Research Practice Guidelines. Arch Gen Psychiatry. 1998;55(12):1121-7.

4. Owens DK, Lohr KN, Atkins D, Treadwell JR, Reston JT, Bass EB, et al. AHRQ series paper 5: grading the strength of a body of evidence when comparing medical interventions-agency for healthcare research and quality and the effective health-care program. J Clin Epidemiol. 2010;63(5):513-23.

5. Nelson JC, Papakostas GI. Atypical antipsychotic augmentation in major depressive disorder: a meta-analysis of placebocontrolled randomized trials. Am J Psychiatry. 2009;166(9): 980-91.

6. American Psychiatric Association. Practice guideline for the treatment of patients with major depressive disorder (revision). Am J Psychiatry. 2000;157(4 Suppl.):1-45.

7. American Psychiatric Association. Practice guideline for the treatment of patients with major depressive disorder (revision 3rd edition), 2010. http://www.psychiatryonline.com/pracGuide/prac GuideChapToc_7.

8. Kennedy SH, Lam RW, Parikh SV, Patten SB, Ravindran AV. Canadian Network for Mood and Anxiety Treatments (CANMAT) clinical guidelines for the management of major depressive disorder in adults.Introduction. J Affect Disord. 2009; 117(Suppl. 1):S1-2.

9. National Institute for Health and Clinical Excellence. Depression: the treatment and management of depression in adults. 2009.

10. Anderson IM, Ferrier IN, Baldwin RC, Cowen PJ, Howard L, Lewis $\mathrm{G}$, et al. Evidence-based guidelines for treating depressive disorders with antidepressants: a revision of the 2000 British
Association for Psychopharmacology guidelines. J Psychopharmacol. 2008;22(4):343-96.

11. Trangle MDB, Gabert T, Haight B, Lindvall B, Mitchell J, Novak H, Rich D, Rossmiller D, Setterlund L, Somers K. Institute for Clinical Systems Improvement. Major Depression in Adults in Primary Care. 2010. Accessed Sep 2012.

12. Malhi GS, Adams D, Porter R, Wignall A, Lampe L, O'Connor $\mathrm{N}$, et al. Clinical practice recommendations for depression. Acta Psychiatrica Scandinavica Supplementum. 2009;439:8-26.

13. Nutt DJ, Davidson JR, Gelenberg AJ, Higuchi T, Kanba S, Karamustafalioglu $\mathrm{O}$, et al. International consensus statement on major depressive disorder. J Clin Psychiatry. 2010;71(Suppl. E1): e08.

14. Harter M, Klesse C, Bermejo I, Schneider F, Berger M. Unipolar depression: diagnostic and therapeutic recommendations from the current S3/National Clinical Practice Guideline. Dtsch Arzteblatt Int. 2010;107(40):700-8.

15. Bauer M, Bschor T, Pfennig A, Whybrow PC, Angst J, Versiani M, et al. World Federation of Societies of Biological Psychiatry (WFSBP) guidelines for biological treatment of unipolar depressive disorders in primary care. World J Biol Psychiatry Off J World Fed Soc Biol Psychiatry. 2007;8(2):67-104.

16. American Psychiatric Association. Practice guideline for the treatment of patients with major depressive disorder. 3rd ed. Washington, DC: American Psychiatric Publishing; 2010.

17. Keitner GI. Adding atypical antipsychotics to antidepressants increases response in treatment-resistant major depression but increases discontinuation as a result of adverse events. Evid Based Med. 2010;15(1):19-20.

18. Carpenter LL, Yasmin S, Price LH. A double-blind, placebocontrolled study of antidepressant augmentation with mirtazapine. Biol Psychiatry. 2002;51(2):183-8.

19. Blier P, Ward HE, Tremblay P, Laberge L, Hebert C, Bergeron R. Combination of antidepressant medications from treatment initiation for major depressive disorder: a double-blind randomized study. Am J Psychiatry. 2010;167(3):281-8.

20. Lam RW, Kennedy SH, Grigoriadis S, McIntyre RS, Milev R, Ramasubbu R, et al. Canadian Network for Mood and Anxiety Treatments (CANMAT) clinical guidelines for the management of major depressive disorder in adults. III. Pharmacotherapy. J Affect Disord. 2009;117(Suppl. 1):S26-43.

21. Parikh SV, Segal ZV, Grigoriadis S, Ravindran AV, Kennedy SH, Lam RW, et al. Canadian Network for Mood and Anxiety Treatments (CANMAT) clinical guidelines for the management of major depressive disorder in adults. II. Psychotherapy alone or in combination with antidepressant medication. J Affect Disord. 2009;117(Suppl. 1):S15-25.

22. Thase ME, Trivedi MH, Nelson JC, Fava M, Swanink R, Tran $\mathrm{QV}$, et al. Examining the efficacy of adjunctive aripiprazole in major depressive disorder: a pooled analysis of 2 studies. Prim Care Companion J Clin Psychiatry. 2008;10(6):440-7.

23. El-Khalili N, Joyce M, Atkinson S, Buynak RJ, Datto C, Lindgren $\mathrm{P}$, et al. Extended-release quetiapine fumarate (quetiapine $\mathrm{XR}$ ) as adjunctive therapy in major depressive disorder (MDD) in patients with an inadequate response to ongoing antidepressant treatment: a multicentre, randomized, double-blind, placebo-controlled study. Int J Neuropsychopharmacol. 2010;13(7):917-32.

24. Bauer M, El-Khalili N, Datto C, Szamosi J, Eriksson H. A pooled analysis of two randomised, placebo-controlled studies of extended release quetiapine fumarate adjunctive to antidepressant therapy in patients with major depressive disorder. J Affect Disord. 2010;127(1-3):19-30.

25. Papakostas GI. Augmentation strategies in the treatment of major depressive disorder. Examining the evidence on augmentation with atypical antipsychotics. CNS Spectr. 2007;12(12 Suppl. 22): $10-2$. 
26. Papakostas GI, Shelton RC, Smith J, Fava M. Augmentation of antidepressants with atypical antipsychotic medications for treatment-resistant major depressive disorder: a meta-analysis. J Clin Psychiatry. 2007;68(6):826-31.

27. Karamustafalioglu O. Major depressive disorder, mental health care, and the use of guidelines in the Middle East. J Clin Psychiatry. 2010;71(Suppl. E1):e07.

28. Davidson JR. Major depressive disorder treatment guidelines in America and Europe. J Clin Psychiatry. 2010;71(Suppl. E1):e04.

29. Higuchi T. Major depressive disorder treatment guidelines in Japan. J Clin Psychiatry. 2010;71(Suppl. E1):e05.

30. Zhang M. Major depressive disorder treatment guidelines in China. J Clin Psychiatry. 2010;71(Suppl. E1):e06.

31. Trivedi MH, Rush AJ, Crismon ML, Kashner TM, Toprac MG, Carmody TJ, et al. Clinical results for patients with major depressive disorder in the Texas Medication Algorithm Project. Arch Gen Psychiatry. 2004;61(7):669-80.

32. Suehs BTAT, Bendele SD, Crismon ML, Trivedi MH, Kurian B. Texas Medication Algorithm Project procedural manual: Major depressive disorder algorithms. Austin, TX: Texas Department of
State Health Services; 2008. http://www.dshs.state.tx.us/mhpro grams/disclaimer.shtm. Accessed 20 Aug 2012.

33. Yoshino A, Sawamura T, Kobayashi N, Kurauchi S, Matsumoto A, Nomura S. Algorithm-guided treatment versus treatment as usual for major depression. Psychiatry Clin Neurosci. 2009;63(5): $652-7$.

34. Katon W, Von Korff M, Lin E, Simon G, Walker E, Unutzer J, et al. Stepped collaborative care for primary care patients with persistent symptoms of depression: a randomized trial. Arch Gen Psychiatry. 1999;56(12):1109-15.

35. Unutzer J, Katon W, Callahan CM, Williams JW Jr, Hunkeler E, Harpole L, et al. Collaborative care management of late-life depression in the primary care setting: a randomized controlled trial. JAMA. 2002;288(22):2836-45.

36. Gonzalez HM, Vega WA, Williams DR, Tarraf W, West BT, Neighbors HW. Depression care in the United States: too little for too few. Arch Gen Psychiatry. 2010;67(1):37-46.

37. Grol R. Successes and failures in the implementation of evidencebased guidelines for clinical practice. Med Care. 2001;39(8 Suppl. 2):II46-54. 